REVISTA CIENCIAS BIOMÉDICAS

ARTÍCULOS ORIGINALES

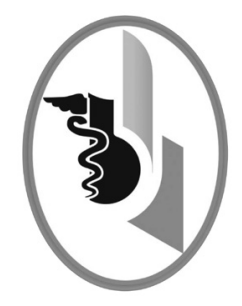

\title{
MORBILIDAD MATERNA EXTREMA EN UNA CLÍNICA OBSTÉTRICA COLOMBIANA*
}

\author{
EXTREME MATERNAL MORBIDITY IN A COLOMBIAN \\ OBSTETRIC CLINIC
}

Rivas-Perdomo Edgar ${ }^{1}$

Vásquez-Deuloffeut Doris ${ }^{2}$

Correspondencia: erivasperdomo@gmail.com

Recibido para evaluación: marzo - 25 - 2014. Aceptado para publicación: mayo - 21- 2016.

\section{RESUMEN}

Introducción: la enfermedad y la mortalidad materna son un problema de salud pública altamente complejo, determinado por las condiciones individuales de la mujer, la calidad de los servicios médicos y los contextos culturales y sociales.

Objetivo: realizar evaluación retrospectiva de casos con morbilidad materna extrema (MME) en una clínica de atención obstétrica que cumple actividades universitarias.

Materiales y métodos: estudio de corte transversal realizado con la información estadística arrojada luego de la atención de las pacientes obstétricas atendidas en la Clínica de Maternidad Rafael Calvo (CMRC) en Cartagena, con criterios para definición de morbilidad materna extrema durante el año 2014. Se consideraron variables como la edad de la paciente, paridad, edad gestacional,y se estimó la causa de la MME. Se calcularon los indicadores: razón de mortalidad materna, razón de morbilidad materna extrema, índice de mortalidad, relación MME/MM, relación criterio/caso, porcentaje de MME e índice de mortalidad perinatal. Se utilizó el software Stata-12.

Resultados: se identificaron 625 casos con criterios de MME. Edad promedio de 23.9 años. El $42.5 \%$ no realizaron control prenatal. Edad gestacional 31.0 semanas. Los trastornos hipertensivos son las causas más frecuentes de MME seguidas de las hemorragias y sepsis. Razón de mortalidad materna 11.5/1000 nv, razón de MMEG: 71.6, índice de mortalidad: $0.2 \%$, relación MME/MM: 625 , relación criterio/ caso: $2.6 \%$, casos MME con 3 criterios: 40.2 e índice de mortalidad perinatal por MME: 9.6.

Conclusión: la razón de morbilidad materna extrema fue de $71.6 \times 1000$ nv, con relación $\mathrm{MME} / \mathrm{mm}$ de 625 . Una relación criterio/caso: 2.6 y $40.2 \%$ de los casos con tres o más criterios de inclusión. Rev.cienc.biomed. 2016;7(1):54-61.

\section{PALABRAS CLAVE}

Morbilidad; Preeclampsia; Sepsis.

* Presentado en XIV Congreso Colombiano de Medicina Perinatal. 2015

1 Médico. Especialista en Ginecología y Obstetricia. Docente. Facultad de Ciencias de la Salud. Programa Medicina. Universidad del Magdalena. Santa Marta. Colombia.

2 Enfermera Epidemióloga. Clínica de Maternidad Rafael Calvo. Cartagena. Colombia 


\section{SUMMARY}

Introduction: the illness and maternal mortality are a public health problem very complicated, determined by individual conditions of the woman and the quality of public medical services as well as cultural and social contexts.

Objective: to carry out a retrospective evaluation about extreme maternal morbidity cases in an obstetric clinic which perform university activities.

Methods: transversal study carried out with statistic information obtained after the care of obstetric patients that consulted the Clinica Maternidad Rafael Calvo (CMRC) with criteria to define extreme maternal morbidity during 2014. It was considered variables like age of patient, parity, gestational age and extreme maternal morbidity cause. Also, it was calculated: maternal mortality reason, extreme maternal morbidity reason, mortality index, extreme maternal morbidity/ maternal mortality relation, criterion/case relation, extreme maternal morbidity percentage and perinatal mortality index. Stata-12 software was carried out.

Results: 625 cases were identified with criteria of extreme maternal morbidity. Average age of 23.9 . The $42.5 \%$ did not carry out prenatal control. Average of gestational age of 31.0. Hypertensive disorders are the most frequent causes of extreme maternal morbidity followed by hemorrhages and sepsis. Maternal mortality reason: $11.5 / 1000$ nv, criterion/case relation: $2.6 \%$, extreme maternal morbidity cases with 3 criteria: 40.2 and perinatal mortality index by extreme maternal morbidity: 9.6.

Conlusion: the reason of extreme maternal morbidity was $71.6 \times 1000$ nv with EMM/ MM relation of 625 . Criterion/case relation: 2.6 y $40.2 \%$ in cases with three or more inclusion criteria. Rev.cienc.biomed. 2016;7(1):54-61.

\section{KEYWORDS}

Morbidity; Pre-Eclampsia; Sepsis.

\section{INTRODUCCIÓN}

La morbimortalidad materna es un problema complejo de salud pública, determinado tanto por las condiciones individuales de la mujer, la calidad de los servicios médicos, así como por los contextos culturales y sociales. El reconocimiento de la carga generada por las complicaciones de la gestación ha dado lugar al concepto de morbilidad materna extrema (MME), entendido como el caso en el cual ocurre disfunción orgánica aguda que, si no es tratada adecuadamente, podría resultar en muerte materna $(1,2)$. Es una complicación obstétrica severa que requiere intervención médica urgente para impedir el posible fallecimiento de la madre durante el embarazo, el parto o dentro de los 42 días siguientes a la terminación del embarazo $(3,4)$.

El análisis de las morbilidades maternas extremas "near miss" provee más información de los riesgos a los que las madres pueden estar expuestas durante el embarazo, que los análisis realizados a los casos de muerte materna (5), es decir, la integración del estudio de la morbilidad ma- terna extrema al de la mortalidad materna incrementa la oportunidad de identificar los factores que contribuyen a ese continuum que va desde el embarazo sin complicaciones hasta la muerte (6). Su análisis aporta información directa acerca de los dilemas diagnósticos y de tratamiento (7), y, ayuda a identificar el patrón de apoyo intensivo necesario para las mujeres gestantes con complicaciones graves que llegan al centro de salud (8).

Se estima que la prevalencia mundial de complicaciones que amenazan potencialmente la vida de la mujer gestante varía entre 0.3 y 101.7 casos por cada 1.000 nacidos vivos, con una media de 8.2 casos por 1.000 nacidos vivos (9). La vigilancia de la MME es una de las estrategias propuestas dentro de las acciones regionales de OPS/OMS para mejorar la salud materna y lograr reducir la tasa de mortalidad materna (uno de los objetivos de desarrollo del milenio) $(10,11)$. La intención del estudio es realizar evaluación retrospectiva de los casos de MME, en una clínica obstétrica donde se cumplen actividades educativas universitarias. 


\section{MATERIALES Y MÉTODOS}

Estudio de corte transversal realizado con la información estadística arrojada luego de la atención de las pacientes obstétricas atendidas en la Clínica de Maternidad Rafael Calvo (CMRC) en Cartagena durante el año 2014. Se tomaron los casos con criterios para ser considerados casos de $M M E$, según los parámetros incluidos en el protocolo de vigilancia en salud para MME del Instituto Nacional de Salud de Colombia. Se consideraron variables demográficas como la edad de la paciente, paridad, edad gestacional y se estimó la causa de la MME.

Se calcularon los valores totales de los siguientes eventos. Nacidos vivos (NV), muertes maternas (MM), mortalidad perinatal, morbilidad materna extremadamente grave (MME), total criterios de inclusión casos de MME y casos MME con tres o más criterios de inclusión. Además, los siguientes indicadores: razón de mortalidad materna, que hace referencia al número de casos de $\mathrm{MME} /$ número de nacidos vivos dentro del mismo período $X$ 1.000. Índice de mortalidad, indica letalidad en las mujeres con MME. Número de casos de muertes maternas certificadas en el período/número de casos por muertes maternas más número de MME certificadas en el mismo período. Relación MME/MM, número de casos de $\mathrm{MME} /$ número de casos por muertes maternas tempranas. Relación criterio/caso: es la sumatoria de criterios de inclusión relacionados en los casos según la causa principal certificada en un período/ número de MME asociados a la causa principal certificados en el mismo período. Porcentaje de casos de MME con tres o más criterios de inclusión. Índice de mortalidad perinatal por $M M E$, número de muertes perinatales de casos $\mathrm{MME} /$ número de casos de MME X 100.

Además, se determinó la frecuencia de las consultas prenatales y la semana de gestación en la que se iniciaban. Se identificó el grupo de edad gestacional más frecuentemente afectado de MME, así como la ubicación de la MME con respecto al parto. Se identificaron los resultados perinatales.
Para el análisis se utilizó el software estadístico Stata-12. Se describieron las variables continuas por medio de medidas de tendencia central y de dispersión; las variables categóricas se presentan por medio de proporciones.

Aspectos éticos: es una investigación sin riesgo, de acuerdo con la Resolución 8430 de 1993 del Ministerio de Salud y Protección Social de la República de Colombia y la Declaración de Helsinki de la Asociación Médica Mundial. Se contó con la aprobación del Comité de Ética de la Institución y se preservó la confidencialidad.

\section{RESULTADOS}

En el año 2014 se registraron 8.725 nacimientos vivos en la CMRC. Se identificaron 625 casos que cumplían criterios de MME. Edad materna promedio 23.9 años con extremos entre 13 y 45 años; el $50 \%$ en el intervalo central y los q25-75 fueron 19 y 28 años de edad (Figura No 1).

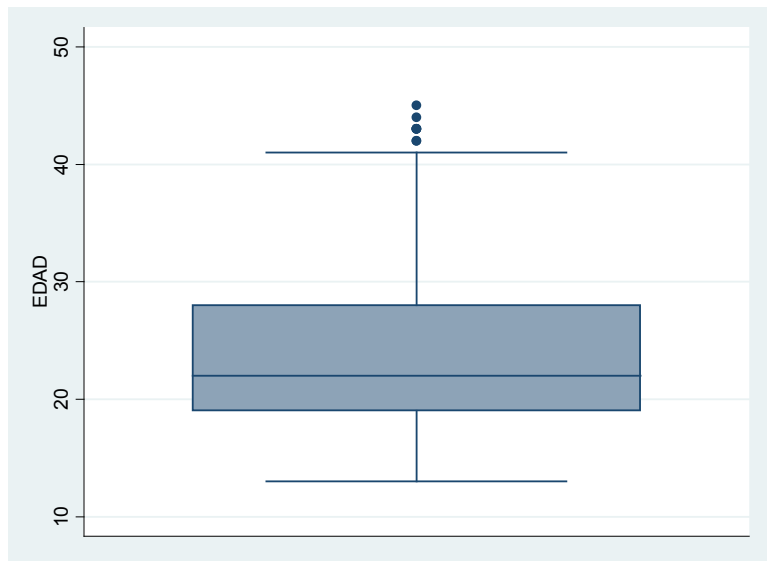

Figura $\mathbf{N}^{\circ} 1$.

Casos de MME. Medidas de tendencia central y dispersión de la edad materna

El q25-75 del número de gestaciones fue 2-3 en los valores extremos de uno a diez embarazos. El $81.8 \%$ de las pacientes había tenido tres o menos gestaciones: $39.2 \%$ primigravidas; $6.0 \%$ con dos gestaciones y $16.5 \%$ con tres gestaciones.

La paridad tuvo extremos entre uno y trece hijos. El q25-75 fue entre uno y dos hijos. El $33.4 \%$ de las pacientes había tenido al me- 
nos un aborto, dos pacientes informaron antecedente de enfermedad trofoblástica gestacional y 25 habían presentado embarazo ectópico.

El período intergenésico tuvo extremos de cero a 19 años; en el $50 \%$ de la población fue entre cero y dos años. El $42.5 \%$ de las pacientes no realizaron control prenatal. El $47.4 \%$ restante tuvo media de cinco consultas prenatales, para q25-75 de 4-6 visitas y valores extremos de uno a 16 controles. La edad gestacional promedio al momento del evento de MME fue 31.0 semanas con valores extremos de 3 a 43 y q25-75: de 27 a 38 semanas de embarazo.

El $65.2 \%$ de la MME ocurrió intraparto, 19.6\% en posparto y $15.1 \%$ anteparto. El $52.0 \%$ de los casos de MME se finalizó la gestación por cesárea y el $24.3 \%$ por parto vaginal. En el $12.8 \%$ de los casos el embarazo continuó su curso y en el $10.8 \%$ fueron abortos.

Resultados perinatales: se registraron 477 nacimientos, de los cuales 417 (66.7\%) fueron productos vivos al nacer, y 60 casos $(9.6 \%)$ correspondieron a muertes fetales. Los pesos observados (de los cuales 9 correspondían a embarazos gemelares) concentraron el $50 \%$ de los valores centrales entre 2.030 y 3.200 gramos con valores extremos: 500 y 5.601 gramos.
El 34\% de las muertes fetales se relacionó con trastornos hipertensivos del embarazo, el $22 \%$ fue por insuficiencia placentaria y el $15 \%$ por hipoxia intrauterina. En el $10 \%$ de la muerte fetal se asoció a desprendimiento prematuro de placenta.

Los indicadores de vigilancia en MME se presentan en la Tabla No 1 . A su vez, la Tabla $\mathrm{N}^{\circ} 2$ presenta la MME distribuida por grupos etarios.

\begin{tabular}{|l|c|c|c|}
\hline \multicolumn{4}{|c|}{ Tabla No 2.} \\
MME distribución por grupos etarios \\
\hline Grupos etarios & Neto & $\%$ & $\begin{array}{c}\text { Razón x } \\
1000 ~ N V\end{array}$ \\
\hline 10 a 14 años & 8 & 1.28 & 0.9 \\
\hline 15 a 19 años & 164 & 26.2 & 18.8 \\
\hline 20 a 34 años & 394 & 63.0 & 45.1 \\
\hline 35 a 39 años & 47 & 7.5 & 5.3 \\
\hline 40 y mas años & 12 & 1.9 & 1.3 \\
\hline
\end{tabular}

La MME distribuida según causas y agrupada por grupos etarios se presenta en la Tabla $\mathrm{N}^{\circ} 3$. Algunas pacientes presentaron más de una condición clínica por lo que el valor total es mayor del número de pacientes atendidas. Las entidades preexistentes más frecuentemente asociadas a MME fueron anemia de

\begin{tabular}{|l|c|c|}
\hline \multicolumn{2}{|c|}{ Indicadores de vigilancia en MME } \\
\hline \multicolumn{1}{|c|}{ Indicadores } & Resultados & Metas \\
\hline Nacidos vivos (NV) & 8.725 & - \\
\hline Muertes maternas (MM) & 1 & - \\
\hline Mortalidad perinatal & 60 & - \\
\hline Morbilidad materna extremadamente grave (MMEG) & 625 & - \\
\hline Total criterios de inclusión casos de MMEG & 1.619 & - \\
\hline Casos MMEG con tres o más criterios de inclusión & 251 & - \\
\hline Razón de mortalidad materna (x100.000 NV) & 11.5 & $<30$ \\
\hline Razón de MMEG (x1.000 NV) & 71.6 & $<8$ \\
\hline Índice de mortalidad & $0.2 \%$ & $<4$ \\
\hline Relación MMEG/MM & 625 & $>35$ \\
\hline Relación criterios/caso & 2.6 & $<5$ \\
\hline \% de casos de MMEG con 3 o más criterios de inclusión & 40.2 & - \\
\hline Índice de mortalidad perinatal por MME & 9.6 & - \\
\hline
\end{tabular}


Tabla N॰3.

MME según causas y agrupada por grupos etarios. $\mathbf{n}(\%)$

\begin{tabular}{|l|c|c|c|c|c|}
\hline Causa agrupada & 10 a 14 años & 15 a 19 años & 20 a 34 años & 35 a 39 años & 40 y más años \\
\hline $\begin{array}{l}\text { Trastornos } \\
\text { hipertensivos en } \\
\text { el embarazo }\end{array}$ & $6(0.96)$ & $82(13.12)$ & $217(34.72)$ & $31(4.96)$ & $7(1.12)$ \\
\hline $\begin{array}{l}\text { Complicaciones } \\
\text { hemorrágicas }\end{array}$ & $1(0.16)$ & $36(5.76)$ & $89(14.24)$ & $9(1.44)$ & $2(0.32)$ \\
\hline $\begin{array}{l}\text { Complicaciones } \\
\text { del aborto }\end{array}$ & $0(0.0)$ & $6(0.96)$ & $28(4.48)$ & $4(0.64)$ & $3(0.48)$ \\
\hline $\begin{array}{l}\text { Sepsis de origen } \\
\text { obstétrico }\end{array}$ & $1(0.16)$ & $2(0.32)$ & $2(0.32)$ & $1(0.16)$ & $0(.00)$ \\
\hline $\begin{array}{l}\text { Sepsis de origen } \\
\text { no obstétrico }\end{array}$ & $0(0.00)$ & $27(4.32)$ & $37(5.92)$ & $1(0.16)$ & $0(0.00)$ \\
\hline $\begin{array}{l}\text { Sepsis de origen } \\
\text { pulmonar }\end{array}$ & $0(0.00)$ & $3(0.48)$ & $4(0.64)$ & $0(0.00)$ & $0(0.00)$ \\
\hline $\begin{array}{l}\text { Enfermedades } \\
\text { preexistentes }\end{array}$ & $0(0.00)$ & $2(0.32)$ & $9(1.44)$ & $1(0.16)$ & $0(0.00)$ \\
\hline Otras & $0(0.00)$ & $6(0.96)$ & $8(1.28)$ & $0(0.00)$ & $0(0.00)$ \\
\hline
\end{tabular}

células falciformes, síndrome convulsivo, tormenta tiroidea, anemia ferropénica severa y trombocitopenia. La Tabla $\mathrm{N}^{\circ} 4$ presenta los criterios de inclusión de pacientes con MME.

\section{DISCUSIÓN}

La edad promedio de $23.9 \pm 6.5$ años fue comparable con lo reportado por otros autores $(1,11,12)$. Los rangos de edades fueron muy discordantes, por lo que eran muy similares a unos (1,11-13), y menores a los reportados por otros $(2,14-16)$.

La cantidad de gestantes con edad igual o mayor a 35 años fue menor a la encontrada por Oliveira (17). Los grupos de edades extremas en la vida reproductiva mostraron menores proporciones de casos, estos embarazos se suelen considerar de mayor riesgo, sin embargo, el problema sería principalmente en la adolescencia temprana de 10-14 años (17). Se consideró que un embarazo, tomando en consideración solamente la edad de la paciente, no debería presentar mayor tasa de complicaciones.

\begin{tabular}{|l|l|c|c|}
\hline \multirow{4}{|c}{\begin{tabular}{c} 
Tabla No4. \\
\multirow{2}{*}{ Criterios de inclusión de pacientes con MME }
\end{tabular}} \\
\hline \multirow{2}{*}{ Criterios } & \multicolumn{1}{|c|}{ Categoría } & Sí & $\%$ \\
\hline \multirow{2}{*}{$\begin{array}{l}\text { Relacionado } \\
\text { con } \\
\text { enfermedad } \\
\text { específica }\end{array}$} & Eclampsia & 13 & 2.1 \\
\cline { 2 - 4 } & Choque séptico & 45 & 7.2 \\
\cline { 2 - 4 } & $\begin{array}{l}\text { Choque } \\
\text { hipovolémico }\end{array}$ & 95 & 15.2 \\
\hline \multirow{4}{*}{$\begin{array}{l}\text { Relación con } \\
\text { disfunción } \\
\text { orgánica }\end{array}$} & Falla cardiaca & 90 & 14.4 \\
\cline { 2 - 4 } & Falla vascular & 461 & 73.8 \\
\cline { 2 - 4 } & Falla renal & 121 & 19.4 \\
\cline { 2 - 4 } & Falla hepática & 189 & 30.2 \\
\cline { 2 - 4 } & Falla metabólica & 25 & 4.0 \\
\cline { 2 - 4 } & Falla cerebral & 27 & 4.3 \\
\cline { 2 - 4 } & Falla respiratoria & 84 & 13.4 \\
\cline { 2 - 4 } & Falla coagulación & 148 & 23.7 \\
\hline \multirow{3}{*}{$\begin{array}{l}\text { Relacionado } \\
\text { con el } \\
\text { manejo }\end{array}$} & Necesidad de UCI & 117 & 18.7 \\
\cline { 2 - 4 } & $\begin{array}{l}\text { Necesidad de } \\
\text { cirugía adicional }\end{array}$ & 151 & 24.2 \\
\cline { 2 - 4 } & $\begin{array}{l}\text { Necesidad de } \\
\text { transfusión }\end{array}$ & 53 & 8.5 \\
\hline
\end{tabular}


La frecuencia de pacientes primigestantes de esta serie (39.2\%) fue similar al 35\% reportado por Bello-Álvarez (11) en otra clínica de la ciudad. Sin embargo, las frecuencias porcentuales de la paridad fueron disímiles a otros autores $(11,18,19)$. Se estima que la paridad elevada (cuatro o más embarazos) aumenta al doble el riesgo de muerte fetal intraparto (20). La frecuencia de abortos estuvo en concordancia con otros autores (16). En esta serie el evento de MME se presentó más comúnmente durante el parto, lo cual contrasta con otros autores. Por otro lado, Álvarez Toste (21) lo presenta durante el parto en el $13 \%$ de los casos y posparto en el $65.2 \%$. En esta serie se registró durante el parto en el $65 \%$ y el $19.6 \%$ en posparto.

El número de controles prenatales fue similar a lo reportado por Souza en el año 2007 para América Latina y el Caribe en la década de los 90, con una mediana entre 4.7 y 6.6 (22). El $43 \%$ de las pacientes no habían asistido a control prenatal, muy superior a lo reportado por otros autores (16-18). Ello denota el poco avance logrado en la atención prenatal en lo referente al número total de consultas realizadas durante ese período de gestación. Sin embargo, se observa una tendencia a mejorar el momento de inicio que en promedio es a las cinco semanas. Souza encontró inicio en Colombia a los tres meses de embarazo (22).

La atención prenatal brinda el espacio para prestar asistencia integral a la gestante, en la que es necesario identificar los riesgos presentes y establecer planes que incrementen las opciones para que el parto tenga lugar en condiciones de seguridad (21). Las mujeres hispanas son más dadas a tener un CPN inadecuado, lo que podría contribuir a resultados obstétricos adversos (5). El número de consultas ideales no ha sido totalmente aceptado y existe controversia; la OMS propone un modelo de cuatro visitas (23). Pacheco estimó en su estudio un número menor de seis como un factor de riesgo para morbilidad materna extrema (24). Se estima que en Colombia no resultaría prudente reducir el número de citas de control prenatal a menos que se cuente con una monitorización muy cuidadosa del feto y la madre, lo cual es posible solo en grandes ciudades y con pacientes seleccionados (25). Usualmente solo se tienen en cuenta la cantidad de consultas, pero suelen abordarse los contenidos y la calidad médica, educativa y asistencial de la atención prenatal. En el presente estudio el $24 \%$ de las pacientes con MME registraron menos de cuatro consultas prenatales.

No hay acuerdo acerca del período intergenésico ideal. Con base en las opiniones de expertos, la OMS recomendó como seguro el período de 24 meses (20). El período intergenésico encontrado fue menor a lo encontrado por otros autores (18).

La edad gestacional de 31.0 semanas, en la cual en promedio se registraron las complicaciones severas de las gestaciones con $M M E$, es igual a lo reportado por Mariño (18). O'Malley et al. compararon un grupo de pacientes con MME con otro grupo sin criterios de MME y no encontraron diferencias en las edades de los mismos, lo cual sugiere que se trata de un hecho fortuito (26).

En el $75.7 \%$ de los casos se terminó el embarazo. O'Malley et al. (26) indicaron que en su serie el $49 \%$ de los casos requirieron la terminación del embarazo como medida terapéutica. Un número importante (12.8\%) de las pacientes continuaron con el embarazo una vez superaron la alteración sufrida. Ello es importante porque si continúa un embarazo de alto riesgo se debe garantizar una atención integral y adecuada en aras de garantizar la salud sexual y reproductiva de la paciente.

La mayoría de las causas por muerte fetal respondían a cuadros primarios de hipoxia fetal extrínseca, principalmente por alteraciones placentarias (34.0\%), similar a lo encontrado en otros estudios de muerte fetal $(27,28)$. El riesgo de muerte fetal es cuatro veces mayor en pacientes con "near miss". Los resultados perinatales adversos como bajo peso, prematuridad y muerte perinatal con frecuencia acompañan este diagnóstico (29). Con las otras variables encontradas como corioamnionitis, RPM, etc., no fue posible realizar algún análisis por las pequeñas proporciones encontradas. 
La incidencia de trastornos hipertensivos del embarazo en esta serie fue de $54.8 \%$, lo cual es mucho menor a lo reportado por otros autores $(13,30)$. No obstante, sigue teniendo un aporte importante a la morbilidad materna que, también fue reportada por otros autores $(2,24)$. Es seguido de las hemorragias con $21.9 \%$ y de las sepsis con $12.5 \%$ y es comparable con lo reportada por otros autores $(2,12$, 18). El grupo etario de adultos jóvenes (2034 años) fue el más frecuente con $45.1 \%$, que podría explicarse por el hecho de ser el grupo que más tiene partos. Sin embargo, el grupo de adolescentes (19.7\%) fue proporcionalmente mayor al $9.6 \%$ reportado por Vettore (31).

La razón de MME grave es muy superior a los valores esperados $(<8)$ y sobrepasa los valores reportados por otros autores $(2,11,32)$, este indicador evalúa el aporte importante de la morbilidad en la atención de las gestantes en la Institución, lo cual se entiende por tratarse de una institución de referencia. El interés creciente de integrar al estudio de la mortalidad materna al análisis de la morbilidad materna extremadamente grave permite identificar los factores de riesgos presentes en las gestantes, desde un embarazo sin complicaciones hasta la muerte, pues se presenta en un mayor número de casos y, asímismo, con el manejo de aquellos que evolucionaron favorablemente después de la gravedad extrema se pueden evitar nuevos casos de muerte (21). A pesar de eso, el índice de mortalidad ( $0.2 \%)$ se mostró por debajo del valor máximo esperado $(<4)$, y ello se refleja en la relación MME/MM la cual se mostró muy por encima del valor mínimo esperado. Al compararse con un estudio previo de Rojas en el mismo centro se denota mejoría en los estándares de atención a la paciente obstétrica. El indicador de mortalidad perinatal fue de 9.6, cifra que es menor a lo reportado por otros autores (29): letalidad perinatal en las mujeres con MME, evalúa calidad de atención, oportunidad de respuesta para la reducción de la mortalidad fetal y corresponde al porcentaje de muertes fetales por cada cien casos de MME (10) que, en el contexto de morbilidad materna extrema, podría ser un complemento útil en la evaluación de la calidad de la atención (8). Es importante tener en cuenta que el estudio tiene como limitaciones la naturaleza retrospectiva del mismo. No se pudieron evaluar los retrasos en la atención.

\section{CONCLUSIÓN}

Los trastornos hipertensivos del embarazo, la sepsis y las hemorragias fueron las causas más importantes de MME en una clínica obstétrica colombiana. La razón de MME fue de $71.6 \times 1.000 \mathrm{nv}$ con una relación MME/MM de 625,0 . Una relación criterio/caso de 2.6 y $40.2 \%$ de los casos tuvieron tres o más criterios de inclusión.

CONFLICTO DE INTERESES: ninguno que declarar.

FINANCIACIÓN: recursos propios de los autores.

\section{REFERENCIAS BIBLIOGRÁFICAS}

1. González-Ortiz LD, Gómez-Arias RD, Vélez-Álvarez GA, Agudelo-Londoño SM, Gómez-Dávila J, Wylie J. Características de la atención hospitalaria y su relación con la morbilidad materna extrema en Medellín. Colombia. Rev. Panam Salud Pública. 2014;35(1):15-22.

2. Amaral E; Souza JP; Surita F; Luz G; Souza MH; Cecatti J et al. A population-based surveillance study on severe acute maternal morbidity (near-miss) and adverse perinatal outcomes in Campinas, Brazil: The Vigimoma Project a population-based surveillance study on severe acute maternal morbidity (near-miss) and adverse perinatal outcomes in Campinas, Brazil: The Vigimoma Project BMC Pregnancy and Childbirth 2011, 11:9.

3. Goffman D, Madden RC, Harrison EA, Merkatz IR, Chazotte C. Predictors of maternal mortality and near-miss maternal morbidity. J Perinatol. 2007; 27(10):597-601.

4. Roopa PS, Shailja V, Lavanya R, Pratap K, Murlidhar V P, Jyothi S. "Near Miss" obstetric events and maternal deaths in a tertiary care hospital: an audit. J Pregnancy. 2013; http://dx.doi. org/10.1155/2013/393758.

5. Brown H, Small M, Taylor YJ, Chireau M, Howard DL. Near miss maternal mortality in a multiethnic population. Ann Epidemiol 2011;21:73-77. 
6. Zuleta JJ. La morbilidad materna extrema: una oportunidad para aprender de experiencias exitosas en el manejo de la complicación obstétrica. (Edt) Rev. Colomb Obstet Ginecol. 2007; 58: 180-181.

7. Franco-Yáñez CE, Hernández-Pacheco JA. Monitoreo de morbilidad materna extrema (near miss) como compromiso internacional para complementar la calidad de la atención en salud materna. Perinatol Reprod Hum. 2016;30(1):31-38.

8. World Health Organization. Evaluating the quality of care for severe pregnancy complications: the WHO near-miss approach for maternal health. 2011.

9. Quintero J, Olaya S, Peña A, Gil Y, Palencia Y. Morbilidad materna extrema (MME): descripción de una serie de 23 casos y análisis de las "tres demoras". RFS - Revista Facultad de Salud. 2013; 5 (2): 52-60

10. Instituto Nacional de Salud. Protocolo de vigilancia en salud pública. Morbilidad materna extrema. Bogotá. 2014.

11. Bello-Álvarez L, Vásquez-Deulofeutt D, Rojas-Suarez J, Sará-Fortich P, Cogollo-González M, Jaramillo J. et al. Indicadores de morbilidad materna extrema en una clínica universitaria de tercer nivel de complejidad. Evaluación retrospectiva. Rev.cienc.biomed.2012; 3(2):291-299.

12. Rojas JA, Cogollo M, Miranda JE, Ramos E, Fernández JC, Bello A. Morbilidad materna extrema en cuidados intensivos obstétricos. Cartagena (Colombia) 2006 - 2008.Rev Colomb Obstet Ginecol. 2011;62( 2):131-140.

13. Reisdorfer S; Madi J; Rombaldi RL; Fauth de Araújo B; Barazzetti D; Pavan G. et al. Características clínicas de pacientes obstétricas admitidas en una unidad de tratamento intensivo Terciária: revisão de dez anos. Revista da amriGs, porto alegre, 2013;57(1):26-30.

14. Donati S, Senatore S, Ronconi A \& the regional maternal mortality working Group. Obstetric near-miss cases among women admitted to intensive care units in Italy. Acta Obstet Gynecol Scand 2012; 91:452-457.

15. Villalba-Toquica C; Martínez-Silva P. Morbilidad materna extrema en la clínica universitaria Colombia, Bogotá (Colombia), 2012-2013. Rev Colomb Obstet Ginecol. 2014; 65: 290-296.

16. Paes L; Alvim-Pereira F; Menezes de Mendonça C; Menezes F; Góis K; Farias R. et al. The prevalence of severe maternal morbidity and near miss and associated factors in Sergipe, Northeast Brazil. BMC Pregnancy and Childbirth 2014, 14:25 doi:10.1186/1471-2393-14-25.

17. Oliveira Jr F; Garanhani F; Pinto e Silva JL; Cecatti JG; Parpinelli MA; Haddad SM; et al. Severe maternal morbidity and maternal near miss in the extremes of reproductive age: results from a national cross-sectional multicenter study. BMC Pregnancy and Childbirth 2014,14:77. doi:10.1186/1471-2393-14-77.

18. Mariño C; Vargas D. Caracterización de la morbilidad materna extrema en el Instituto Materno Infantil - Hospital La Victoria. Tesis. Universidad Nacional de Colombia. Bogotá. 2010.

19. Acosta I; Leal Y; Charchalac A; Carrillo L. Caracterización clínica y vigilancia epidemiológica de la morbilidad materna severa. Tesis. Universidad de San Carlos de Guatemala. Centro Universitario de Oriente. Chiquimula, Guatemala. Agosto 2014.

20. Fescina RH, De Mucio B, Díaz Rossello JL, Martínez G, Serruya S, Durán P. Salud sexual y reproductiva: guías para el continuo de atención de la mujer y el recién nacido focalizadas en APS. 3a.edición. Montevideo: CLAP/SMR; 2011.

21. Álvarez Toste M, Salvador Álvarez S, González G, Pérez R. Caracterización de la morbilidad materna extremadamente grave. Revista Cubana de Higiene y Epidemiología, 2010; 48 (3): 310-320.

22. Souza JP; Parpinelli MA; Amaral E; Cecatti JG. Assistência obstétrica e complicações graves da gestação na América Latina e Caribe: análise das informações obtidas a partir de inquéritos demográficos de saúde. Rev Panam Salud Publica/Pan Am J Public Health 2007; 21(6): 396-401.

23. Villar J, Ba'aqeel H, Piaggio G, Lumbiganon P, Belizán JM, Farnot $U$ et al. WHO antenatal care trial research group. WHO antenatal care randomised trial for the evaluation of a new model of routine antenatal care. Lancet 2001;357:1551-64.

24. Pacheco AJ, Katz L, Souza AS, de Amorim MM. Factors associated with severe maternal morbidity and near miss in the Sao Francisco Valley, Brazil: a retrospective, cohort study. BMC Pregnancy and Childbirth 2014, 14:91.

25. Ministerio de Salud y Protección Social - Colciencias. Guía de práctica clínica para la prevención, detección temprana y tratamiento del embarazo, parto o puerperio. Guías No. 11-15. Bogotá. 2013.

26. O'Malley EG, Popivanov P, Fergus A, Tan T, Byrne B. Maternal near miss: what lies beneath? Eur J Obstet Gynecol Reprod Biol 2016; 199 : 116-120120.

27. Rivas-Perdomo E, Vásquez D. Óbito fetal: hallazgos de patología en una institución de alta complejidad. Cartagena, Colombia, 2010-2011. Rev. Colomb Obstet Ginecol. 2012; 63(4):376-81.

28. Rivas-Perdomo E, Vásquez -Deulofeutt D; Méndez-Rodríguez. Abortos tardíos: hallazgos en estudios de anatomía patológica. Rev.cienc.biomed. 2014:5(2):247-252.

29. Costa L, Ribeiro da Costa A. Óbitos fetais e neonatais entre casos de near miss materno. Rev Assoc Med Bras. 2013; 59(5): 487-494.

30. Curiel-Balsera E, Prieto-Palomino MA, Muñoz-Bono J, Ruiz de Elvira MJ, Galeas JL, Quesada García G. Análisis de la morbimortalidad materna de las pacientes con preeclampsia grave, eclampsia y síndrome HELLP que ingresan en una unidad de cuidados intensivos ginecoobstétrica. Med Intensiva. 2011;35(8):478-483.

31. Vettore MV, Dias M, Soares RM, Vettore M, Leal M. Cuidados pré-natais e avaliação do manejo da hipertensão arterial em gestantes do SUS no Município do Rio de Janeiro, Brasil. Cad. Saúde Pública, Rio de Janeiro, 2011; 27(5 mai):1021-1034.

32. Magalhães MC, Bustamante-Teixeira MT. Morbidade materna extremamente grave: uso do Sistema de Informação hospitalar. Rev Saúde Pública 2012; 46(3):472-8. 